ARID International Journal of Educational and Psychological Sciences (AIJEPS) VOL: 3, NO. 5, January 2022

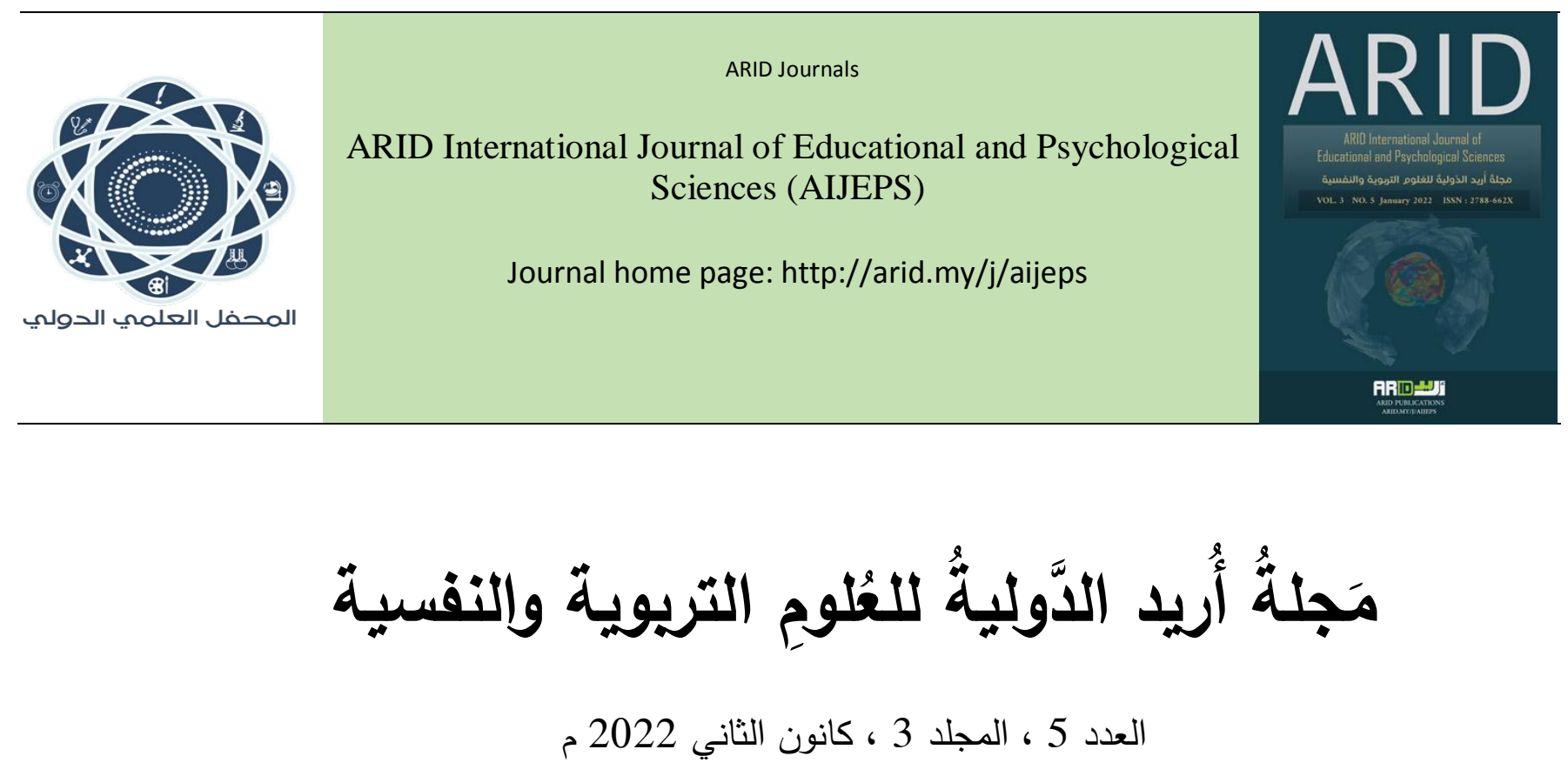

\title{
The relationship between Fear of COVID-19 and Loneliness among Yemeni students in China
}

العلاقة بين مستوى الخوف من كوفيد-19 ودرجة الشعور بالوحدة النفسية لاى الطلبة اليمنيين في الصين

$$
\text { عامر محمد الضبياني }
$$




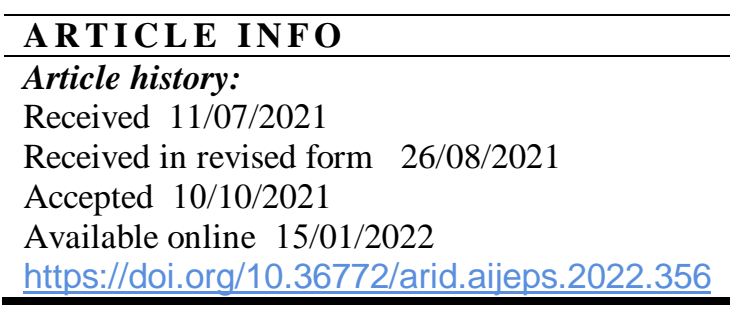

\begin{abstract}
The current research aims to identify the level of fear of Covid-19 and loneliness among Yemeni students in China, and to determine the relationship between them and whether there are differences in the responses of the research sample according to gender. The research adopted the descriptive approach, and the questionnaire was used as a tool for collecting data from the research sample. The research community consisted of Yemeni students in China who were enrolled in the 2021 academic year, the sample is (301) male and female students who were selected using a simple random sampling method. The results showed that the level of fear of Covid-19 and the degree of loneliness were high. There is a positive relationship between fear of COVID-19 and loneliness. There are differences in fear of Covid-19 according to the gender variable in favor of males, and differences in loneliness in favor of females.
\end{abstract}

Keywords: (psychological loneliness, fear of COVID-19, Yemeni students in China. 


\section{الملخص}

يهدف البحث الحالي إلى التعرف إلى مستوى كل من الخوف من كوفيد-19 ودرجة الثعور بالوحدة النفسية لدى الطلبة اليمنيين

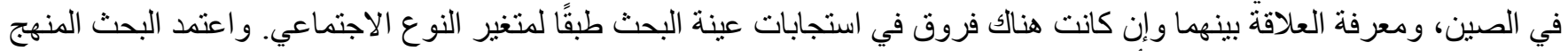

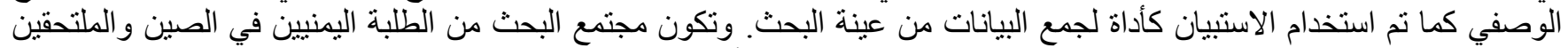

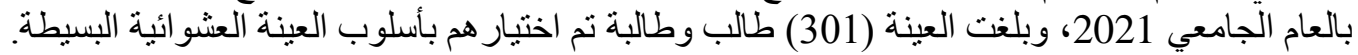

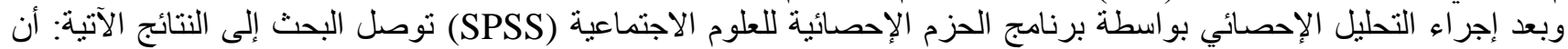

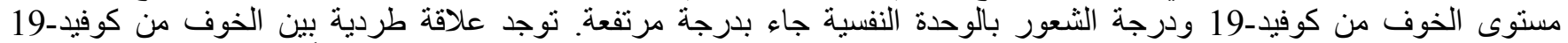

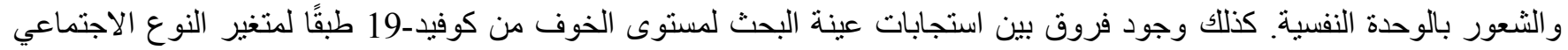
لصالح الذكور، وفروق لارجة الشعور بالوحدة النفسية لصالح الإناث.

الكلمات المفتاحية: (الوحدة النفسية، الخوف من كوفيد-19، الطلبة اليمنيين في الصين) 


\section{المبحث الأول \\ الإطار العام للبحث}

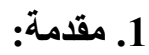

أدى ظهور مرض فيروس كورونـا 2019 (COVID-19) إلى حالة من الذعر في جميع أنحاء العالم خصوصًا مـع ارتفاع عدد الحالات الصصابة وازدياد عدد الوفيات في الصين، والذي ما لبث هذا الأمر حتى أصبح حالة طوارئ على مستوى العالم. كما أدت القيود المطولة على التنقل و الانكماش الاقتصادي في الجائحة إلى الكثير عن التغيرات في المستوى النفسي و التوتر و القلق والاكتئاب ( Wang) Berg-) et al, 2020 بالإضافة إلى العديد من النتائج النفسية السلبية الأخرى مثل الثعور بالوحدة النفسية وعدم الرضـا عن الحياة

.(Weger \& Morley, 2020

و الوحدة النفسية هي مشكلة صحية عامة خطيرة استحوذت على قلق كبير، وتثير إلى تجربة ذاتية أو استجابة غير سارة تتضمن تصورًا ذانيًا للقصور الاجتماعي أو عدم وجود اتصال اجتماعي مع الآخرين (Thamboo, 2016). والوحدة النفسية مؤلمة؛ لأنها تثبير إلى الإقصاء الاجتماعي (Hawkley \& Cacioppo, 2010)، كما أن الوحدة النفسية تعد أحد أقوى عوامل الخطر للنتائج السلبية على الصحة و الرفاهية، بما في ذلك الوفيات (Lindsay, Young, Brown, 2015).

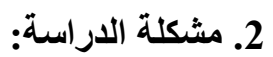

يو اجه طلبة الجامعات عددًا من المشكلات المتعلقة بالصحة العقلية مقارنة بالأفر اد الآخرين من خلفية غير جامعية، ويعاني الطلبة الأجانب في الصين وخصوصًا العرب من ضعف في التو اصل مع المجتمع المحيط بسبب الاختلافات في الثقافة واللغة (لياو جينغ ،

في ديسمبر 2019، بدأ COVID-19 بالانتثار في الصين. في فبراير 2020، طُلب من جميع الطلبة الأجانب في الصين البقاء في جامعاتهم ولم يتمكن أحد من العودة إلى دياره. في معظم الأوقات، يتعين عليهم البقاء في مساكنهم و الاتصـال فقط بأهاليهم وأصدقائهم باستخدام الهو اتف المحمولة. عند الخروج في الهواء الطلق، يجب عليهم الابتعاد عن الآخرين وتغطية أفو اههم و أنوفهم بأقنحة لتجنب العدوى. كان هذا وقتًا يميل فيه الجميع إلى الثـعور بالوحدة وتجربة المشاعر السلبية. وكثفت دراسة سابقة أجراهـا الباحث عن معاناة الطلبة اليمنيين في الصين من عدد من المشكلات النفسية أبرز ها الثعور بالوحدة النفسية (الضبياني، 2021)؛ لذلك يهدف البحث الحالي إلى الإجابة عن السؤال الرئيس: ما العلاقة بين مستوى الخوف من كوفيد 19 ودرجة الثـعور بالوحدة النفسية لدى الطلبة اليمنيين في 


\section{3.}

$$
\text { يهذف البحث الحالي إلى الآتي: }
$$

- التعرف إلى مستوى الخوف من كوفيد-19 لدى الطلبة اليمنيين في الصين. - التعرف إلى درجة الشعور بالوحدة النفسية لدى الطلبة اليمنيين في الصين.

ـ التعرف إلى طبيعة العلاقة بين مستوى الخوف من كوفيد-19 ودرجة الشعور بالوحدة النفسية لدى الطلبة اليمنيين في الصين. - الكثف عما إذا كان هنالك فروق في استجابات عينة البحث طبقًا لمتغير النوع الاجتماعي.

$$
\begin{aligned}
& \text { 4. حدود البحث: } \\
& \text { تتمثل حدود البحث الحالي في الآتي: } \\
& \text { الحدود البشرية: الطلبة اليمنيين الدارسين في الصين. } \\
& \text { الحدود الزمانية: العام الجامعي 2021م. } \\
& \text { الحدود المكانية: الجامعات الصينية. }
\end{aligned}
$$

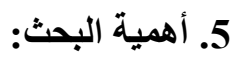

تتمثل أهميـة البحث الحسالي من خـلال تتاولـه لأحد الموضـوعات البحثيـة الهامـة وهو الخوف من كوفيد-19 و علاقة هذا المتغير بالثعور بالوحدة النفسية لاى الطلبة اليمنيين الدر اسين في الصين، خاصةً في الظروف الحالية التي يشهدها العالم في ظل انتشـار فيروس كورونا (كوفيد-19)، كما قد يساعد هذا البحث الجهات ذات العلاقة للتحفيف من مشكلات الطلبة اليمنيين الموفدين للار اسـة في الخـارج لتسليط الضوء على أهم المشكلاًت النفسية التي تواجهوم وتوضيح أسبابها وتقديم بعض التوصيات التي تساهم في الحد منها.

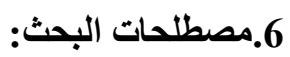
ـالوحدة النفسية (Loneliness): يعرف (الدسـوفي، 1998) الوحدة النفسية بأنها: "حالـة تحدث نتيجـة خلل في شبكة العلاقـات الاجتماعية للفرد سواء كان ذلك في صورة (كمية) لا يوجد عدد كافٍ من الأصدقاء أو في صورة (كيفية) افتقاد المحبة والألفة والتواد من الآخرين". ويعـرف (Lynch, 1977) الثـعور بالوحدة النفسية بأنـه: "حالـة يشـعر فيها الفرد بالوحدة أب بالانفصـال عن الآخـرين ويصاحبها معاناة الفرد لكثير من ضروب الوحشة و الاغتر اب و الاغتمام و الاكتئاب وذلك من جر اء إحساسه بالوحدة". ويعرف الباحث الوحدة النفسية إجر ائيًا بأنها الدرجات التي يحصل عليها الطلبة اليمنيين في الصين محل البحث على مقياس الثعور الوحدة النفسية. 
ـالخوف من كوفيد-19 (Fear of COVID): يعرفه (Arora, Jha, Alat, \& Das, 2020) بأنه استجابة مفرطة للخوف من الإصـابة بـالفيروس المسبب لـ COVID-19، مما يؤدي إلى قلق مفرط مصساحب بشـأن الأعر اض الفسيولوجية، وضغط كبير حول الخسارة الثخصية والمهنية وزيادة الطمأنينة وسلوكيات البحث عن السلامة، وتجنب الأماكن و المواقف العامـة، مما يتسبب في إضعاف ملحوظ في أداء الحياة اليومية. ويعرف الباحث الخوف من كوفيد-19 إجرائيًا بأنها الدرجات التي يحصل عليها الطلبة اليمنيين في الصين محل البحث على مقياس الخوف من كوفيد-19. 7. ـدراسـة الضبياني (2021): و التي هدفت إلى التعرف على درجـة الثـعور بالوحدة النفسية ومستوى كل من القلق و الاكتئاب و إدارة الضغوط و المساندة الاجتماعية لدى الطلبة اليمنيين في الصين، ومعرفة علاقة الوحدة النفسية بتلك المتغير ات و إن كانت هناك فروق في استجابات عينة البحث طبقًا لمتغير النوع الاجتماعي. وتكون مجتمع البحث من الطلبة اليمنيين في الصين و الملتحقين بالعام الجامعي 2019-2020م، وبلغت العينة (509) طالب وطالبة تم اختبار هم بأسلوب العينة العشوائية البسيطة، واعتمد البحث المنهج الوصفي كما تم استخدام الاستبيان كأداة لجمع البيانات من عينة البحث. وتوصل البحث إلى أن الثعور بالوحدة النفسية ومستوى القلق لدى الطلبة اليمنيبن في الصين كانا بدرجة مرتفعة، بينما مستوى بقية المتغيرات (الاكتئاب ـ إدارة الضغوط ـ المسـاندة الاجتماعية) كانت بدرجة متوسطة. كما توجد علاقة طردية بين الثـعور بالوحدة النفسية وكل من القلق و إدارة الضـغوط، والاكتئاب، فيمـا كانت العلاقة عكسية مـع متغير المساندة الاجتماعية. كذلك وجود فروق بين استجابات عينة البحث لدرجة الثعور بالوحدة النفسية ومستوى القلق لصـالح الإنـاث، وفروق في إدارة الضغوط و المساندة الاجتماعيـة طبقًا لمتغير النوع الاجتماعي لصـالح الذكورـ فيما لا توجد فروق بين استجابات عينـة البحث لمستوى الاكتنئاب. ـ دراسة الضبياني وآخرون (2021) و التي هدفت إلى در اسـة العلاقة بين اليقظة العقلية و الرضـا عن الحياة و الوحدة النفسية والتوسط المحتمل للدعم الاجتماعي بين الطلبة الأجانب في الصين. وتكّون مجتمع البحث من الطلبة اليمنيين في الصين والملتحقين بالعام الجامعي 2019-2020م، وبلغت العينة (509) طالب وطالبة نم اختيار هم بأسلوب العينة العشو ائية البسيطة. واعتمد البحث المنهج الوصفي كما تم استخدام الاستبيان كأداة لجمع البيانـات من عينة البحث. وتوصل البحث إلى أن جميع أبعاد اليقظة العقلية الخمسـة تتنبأ بشكل إيجابي بالأبعاد الثلاثة للدعم الاجتماعي، وأن الدعم المتصور من الأصدقاء فقط هو الذي يمكن للمرء أن يشـعر بالرضـا عن الحياة، وفقط الدعم المتصور للآخرين المهمين يمكن أن يقلل من الثعور بالوحدة النفسية. 
ـ دراسة عامر (2021): و التي هدفت إلى تحديد مستويات كل من الخوف من مرض كوفيد -19 و الاكتئاب و الوحدة والضغوط النفسية في المجتمع المصري، و إن كانت هناك فروق في استجابات عينة البحث طبقًا لمتغير النوع الاجتماعي. وتكون مجتمع البحث من (202) فردًا توز عت أعمار هم بين 13 عام و 71 عام. و اعتمد البحث المنهج الوصفي كما تم استخدام الاستبيان كأداة لجمع البيانـات من عينة البحث. وتوصل البحث إلى عدد من النتائج أهمها: أن 74.62٪ من أفراد العينة أبلغوا عن خوف متوسط إلى مرتفع من كوفيد -19، وكذلك لا توجد فروق بين استجابات عينة البحث لمستوى الشعور بالوحدة النفسية. ـ دراسة ناجي (2021): و التي هدفت إلى التعرف إلى مستوى الخوف من وباء Covid-19 بين طلاب الجامعة و إن كانت هناك فروق في استجابات عينة البحث طبقًا لمتغير النوع الاجتماعي. وتكون مجتمع البحث من طلبة جامعة بغداد، وبلغت العينة (200) طالب وطالبة تم اختيار هم بأسلوب العينة العشو ائية البسيطة. واعتمد البحث المنهج الوصفي كما تم استخدام الاستبيان كأداة لجمع البيانات من عينة البحث. وتوصل البحث إلى وجود فروق بين استجابات عينة البحث لمستوى الخوف من وباء Covid-19 لصالح الإناث. المبحث الثاني: الإطار النظري

أولًا: الخوف من كوفيد-19.

يؤثر مرض كوفيد -19 على جميع مجالات الحياة، وتكون عوامل الخطر فريدة من نوعها ومتعددة ومتنوعة أكثر من الأوبئة الأخرى؛ لذلك، قد يمتد الخوف إلى مـا بعد الإصـابة بـالمرض أو المـوت بسبب الإصـابة بالفيروس ويثير مخـاوف أخرى مثل الأزمـة الاقتصادية (Yoon, 2020).

\section{1- 1 مفهوم الخوف من كوفيد-19:}

لا يوجد تعريف دقيق لمفهوم الخوف من كوفيد -19 كونـه مفهومًا جديدًا، ولكن يعرفه (Arora, Jha, Alat, \& Das, 2020) بأنـه استجابة مفرطسة للخوف من الإصـابة بـالفيروس المسبب لـ COVID-19، مدـا يؤدي إلى قلق مفرط مصساحب بشـأن الأعر اض الفسيولوجية، وضغط كبير حول الخسارة الثخصية والمهنية وزيادة الطمأنينة وسلوكيات البحث عن السلامة، وتجنب الأماكن والمو اقف العامة، مما يتسبب في إضعاف ملحوظ في أداء الحياة اليومية. وتتضمن كذلك المحفزات مو اقف أو أشخاصًا تتطوي على احتمال الإصابة بالفيروس، مثل مقابلة أثخاص أو مغادرة المنزل أو السفر أو قراعة التحديثات أو الأخبار أو الإصابة بالمرض أو الذهاب للعمل بالخارج.

\section{2- 2كونات الخوف من كوفيد-19:}

يتضمن الخوف من كوفيد-19 ثلاثة مكونات أساسية تعزز عملية الخوف، هي: - الفسبولوجية: يتم تشغيل استجابة القتال أو الهروب عند التعرض لحدث سـابق. يمكن أن يسبب القلق المستمر أعر اضًا مثنل خفقان القلب، والرعشة، وصعوبة التنفس، والدوخة، وتغير الثهية، والنوم (Wang et al. 2020) ـ 
ـ الإدر اك: الخوف من الفيروس قد ينطوي على الانثغال بالتهديد الذي يثير الإدر اك (Chakraborty \& Chatterjee, 2020) ، على سبيل المثال "سأموت إذا أصبت بالفيروس، لن أتمكن من الذهاب إلى وظيفتي وسأكون عاطلًا عن العمل"؛ "عائلتي في خطر وقد يموتون". قد يؤدي الإدر الك إلى مزيد من الاستجابات العاطفية، مثل الحزن و الشعور بالذنب و الغضب. ـ السلوكية: من أجل منع العو اقب ، ينخرط الأفراد في سلوكيات الأبطال. هناك خوف ملحوظ من استخدام وسائل النقل العام، ولمس أي سطح، والتو اجد في الأماكن المفتوحة (الأسواق، والشواطئ، و الملاعب) وفي الأماكن المغلقة (الفنادق، ومر اكز التسوق، ودور السينما، والملاعب الداخلية)، وحضور أب تجمعات عامة، والوقوف في طابور (Tanner, 2020). يخشى الفرد و يتجنب مو اقف مثل مقابلة الناس أو الإفر اط في الانغماس في سلوكيات السلامة المتعلقة بالصحة (مثل غسل اليدين). تؤدي سلوكيات الطمأنينـة مثل الفحص المستمر لمؤشـرات الجسم الحيويـة، وتأكيد عدم وجـود المرض، و العـلاج الذاتي أو إعـادة فحص الصـرف الصـحي إلى استمرار الخوف (Li et al. 2020)؛ مما يؤدي إلى الرهاب. إلى جانب ذللك، قد يؤدي الأصل الحيو اني للفيروس ( Andersen et) al.2020 إلى مزيد من الخوف من تنـاول أطعمـة تعتمد على اللحوم الغريبـة. على الرغم من أن الخوف و اقعي، إلا أنه يمكن أن يزعج الجودة العامة للأداء اليومي للأفر اد. (Al-Salem, 2021).

\section{3. أسباب الخوف من كوفيد-19.}

تباينت آراء ووجهات النظر حول أسباب الخوف من كوفيد-19 ويرى الباحث أن أهم الأسباب التي أدت إلى الخوف من كوفيد19 الآتي:

- تجاهل القضايا النفسية والاجتماعية مثل الخوف و القلق منذ ظهور مكافحة العدوى و التطعيم ضد COVID-19 حيث لم يؤخذ مرض كوفيد -19 في بداية الأمر على محمل الجد (Orhan, Ömer and Mustafa, 2021). - الخوف من الإصـابة بـ COVID-19. إذا زاد مستوى الخوف من COVID-19، فسيكون من الصـب على الأفراد التصرف بشكل واضح و عقلاني أثناء الاستجابة لكل من COVID-19 و الأحداث الأخرى. - البقاء في المنزل لفترة طويلـة وفرض إجراءات الحجر الصحي تسبب القلق والاكتئاب وتتفاعل طرديًا مـع ارتفـاع مستوى الخوف من الخوف من كوفيد-19). (Madhava; Valsan; Naveen; Krishnakumar,2020). - الانتشار السريع للمعلومات عن طريق وسائل الإعلام، نشأت موجة ذعر عالمية؛؛ مما أدى إلى سلوك غير طبيعي لدى البشر

(Karaka, 2020; Melki, et. al. 2020). 
- أخبار COVID-19 السلبية و غير المتسقة، التي يشـاهدها في الغالب الأشخاص الذين يقيمون في المنزل، على التلفزيون، الإنترنت، ووسـائل التو اصل الاجتمـاعي تجعل الأفر اد متكافئين وأكثر قلقًا ويمكن أن يتسبب في زيادة الرهاب المرتبط بـ

(Daniel; Hall, 2020; Muhammad, 2020). COVID-19 - مشاهدة أفر اد الأسرة أو الأصدقاء المصابين يمكن أن يزيد من النتأثير السلبي لذلك؛ فإن الخوف من COVID-19 سيزيد من

(Salari; Hosseinian-Far; Jalali, الأعر اض النفسية لدى الفرد، ويزيد من الضرر الذي سيحدثه الفيروس للأفر اد 2020).

ثانيًا: الوحدة النفسية.

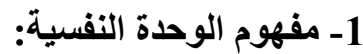

الوحدة من أهم المفـاهيم التـي حظيت باهتمـام كبير في الآونـة الأخيرة مـن قبل البـاحثين في مجـال علم النفس؛ نتيجـة للتغيرات

الاقتصادية و الثقافية والاجتماعية والسياسية التي شهدها المجتمع العالمي في القرن الحادي و العشرين، والتي أدت إلى العديد من المواقف العصيبة على النفس البشرية. وفقًا لديبورا (2018)، تسبب الوحدة مشكلة عقلية وجسدية في حياة الأفر اد إذا استمرت لفترة طويلـة وتزيد من أمر اض القلب و السكتة الدماغية وارتفاع معدل ضغط الدم. علاوة على ذلك، لا يؤثر الشعور بالوحدة على الصحة البدنية فقط بل في (Moeller \& Seehuus, نتائج الحياة السلبية مثل أعر اض الاكتئاب (Cacioppo et al. 2002)، ومشاكل النوم، والتفكير الانتحاري) 2019).

تُعرَّف الوحدة عمومًا بأنها وعي معرفي لنقص الاحتياجات الاجتماعية والثخصية للفرد، وردود الفعل العاطفية للحزن أو الفراغ أو الشوق (Asher \& Paquette, 2003) وفقًا لهينريش وجولون (2006)؛ فإن الثـور بالوحدة في الإنسان هو نتيجـة لتصور هم بأنهم يعتقدون أنهم مختلفون عن الآخرين ويعتقدون أن الناس غير مبالين بهم. الثـعور بالوحدة والوحدة ليسا نفس الثيء. على سبيل المثال، يمكن أن يكون الشخص في اجتمـاع مـع الآخرين و لا يزال يشـعر بالوحدة؛ بينمـا، يمكن أن يكونو ا وحدهم و لا يشـرون بالوحدة حقًا .(Aspel, 2001) ويُعرف (الدسوفي، 1998) الوحدة النفسية بأنها: "حالة تحدث نتيجة خلل في شبكة العلاقات الاجتماعية للفرد سواء كان ذلك في صورة (كمية) لا يوجد عدد كافٍ من الأصدقاء أو في صورة (كيفية) افتقاد المحبة والألفة والتواد من الآخرين" (الدسوقي، 1998: 7). بينما يُعرف سيرمات (Sermat, 1978) الوحدة النفسية بأنها: "عبارة عن الفرق بين أنواع العلاقات الشخصية التي يدرك الفرد أنها لديه في وقت ما، وتلك العلاقات التي يود أن تكون لديه بالاسترشاد بالخبرة السابقة أو بخبرة مثالية لم يسبق له معاينتها في حياته". كما يُعرف 
(Lynch, 1977) من ضروب الوحشة و الاغتر اب والاغتمام والاكتئاب وذلك من جر اء إحساسه بالوحدة" (Lynch, 1977: 233). 2. 2. عناصر الثعور بالوحدة النفسية: تباينت آراء الباحثين حول عناصر الشعور بالوحدة النفسية ويتفق الباحث مـع مـاتتاولته (Rokach,2003) في الأربع العناصر

ـ اغتر اب الذات: وهو شعور الفرد بالفراغ الداخلي والانفصال عن الآخرين واغتر اب الفرد عن نفسه وهويته والحط من قدر الذات. - العزلة في العلاقات الثخصية المنبادلة: ويتمثل ذلك في مشـاعر كون الفرد وحيدًا انفعاليًا وجغر افيًا واجتماعيًا، وشعور الفرد بعدم الانتماء ونقص في العلاقات ذات المعنى لديه حيث يتكون العنصر الآخرين غياب المودة وإدراك الفرد للغياب الاجتمـاعي والثـعور بالخذلان و الهجر.

ـ ألم/ صداع خفيف: وتتمثل في الهياج الداخلي و الثوران الانفعالي للفرد وسر عة الحساسية والغضب وفقدان القدرة على الدفاع والارتباك والاضطر اب واللامبالاة، الذين يستهدف لهم الأفر اد الشاعرون بالوحدة النفسية. - ردود الأفعـال الموجعة الضـاغطة: ويتكون ذلك نتاج مزيج من الألم و المعانـاة والخبرة المعاثـة للشعور بالوحدة النفسية والمتضمنة للاضطر اب و الألم الذي يعايشه الأفر اد الثـاعرين بالوحدة النفسية.

\section{3. أبعاد الشعور بالوحدة النفسية}

حدد (Weiss, 1973) أبعاد أساسية لخبرة الثعور بالوحدة النفسية هي ما أورده (خويطر، 2010: 45): ـ ـ البعد الأول: العاطفة: حيث يحتاج الأفراد دائمًا إلى الصداقة العاطفية الحميمة من الأشخاص المقربين، و إلى التأييد الاجتماعي ويتولد الشعور بالوحدة النفسية نتيجة لفقد الأفراد الشعور بالعاطفة من قبل الآخرين. ـ البعد الثاني: فقدان الأمل (اليأس أو الإحباط): وهو شعور الفرد بالقلق المرتفع والضغط النفسي عند التوقع لاحتياجـات لا تتحقق ممـا يولد الشعور بالوحدة النفسية . ـ البعد الثالث: المظـاهر الاجتماعية: وهي أن شعور الفرد بالوحدة النفسية يقف حائلًا أمسام تكوين الصداقات مع الآخرين، مما يولد الشعور بالاكتئاب، ويجعل الفرد مستهدفًا للإدمان، و انحر اف المر اهقين وسلوكهم سلوكًا يتسم بالعنف و العدوان. 4. - أسباب الثعور بالوحدة النفسية. وللوحدة النفسية أسباب متعددة، بعضها يعود لطبيعة الأشخاص أنفسهر, ويعود البعض الأخر لاضطر ابات كميـة أو كيفية في شكل العلاقات الاجتماعية حيث يـرى كذلك ويس (Weiss,1973) أن الشعور بالوحدة بمكن أن يعزى إلى ما يلي: 
ـ المو اقف الاجتماعيـة (Situational) و هي تركز على النو اقص أو المشكلات و الصـعوبات القائمسة فى البيئة باعتبار هـا أسبابًا مؤديـة للوحدة. ـ الفروق الفردية (Individual) أو ما يعرف بمجمو عة الخصائص الثخصية التي تساعد الأفر اد بالوحدة النفسية منل الخجل و الانطو اء, و العصابية مع وجود اختلافات في الفروق الفردية لدى الأفراد (عطا، 1990). المبحث الثالث: منهجية البحث

1.

اعتمد البحث المنهج الوصفي المسحي كونه الأنسب لهذا النوع من الدراسات الارتباطية.

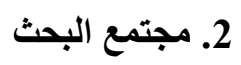

تكون مجتمع البحث من جميع الطلبة اليمنيين الدارسين في الصين و الملتحقين بالعام الجامعي 2021م.

3.

بلغت عينة البحث (301) طالب وطالبة من الطلبة اليمنيين في الصين و الملتحقين بالعام الجامعي 2021م، تم اختيار هم بأسلوب العينة العشو ائية، موزعين حسب النوع الاجتماعي كما هو موضح في الجدول التالي:

جدول(1): توزيع أفر اد عينة البحث طبقًا للنوع الاجتماعي

\begin{tabular}{|c|c|c|}
\hline النسبة & العدد & النوع الاجتماعي \\
\hline$\% 87$ & 262 & ذكر \\
\hline$\% 13$ & 39 & أنثى \\
\hline$\% 100$ & 301 & الإجمالي \\
\hline
\end{tabular}

3.

استخدم الباحث الاستبيان كوسيلة لجمع البيانات من عينة البحث و اعتمد المقاييس الآتية:

ـ مقياس الوحدة النفسية: تم اعتماد المقياس الخاص بالوحدة النفسية والمصمم من قبل جامعـة كاليفورنيـا ( University of California Los Angeles Loneliness Scale)، الـذي أعده كل من رسل، وبيليو، وكترونـا Russel, Peplau, Cutrona) ويتكون من (20) فقرة. تم تعريب و اختبار صدق وثبات المقياس من قبل الأستاذ الدكتور كريم عبد سـاجر خلف الثمري (2012)، كما بلغ معامل ثبات المقياس في هذا البحث (0.91). 
ـ مقياس الخوف مـن كوفيد-19: تم اعتمـاد مقياس الخوف من جائحة كورونـا (COVID-19) في المجتمع العربي. والذي تم تعريبه واختبار صدقه وثباته من قبل الأستاذ الدكتور عبد الناصر السيد عامر (2020)، ويتكون المقياس من (12) فقرة كما بلـغ معامل ثبات المقياس في هذا البحث (0.89).

\section{المبحث الرابع: النتائج والمناقشة}

1. عرض نتائج السؤال الأول ومناقشتها. وللإجابة على السؤال الأول من أسئلة البحث الذي نصه: ما مستوى الخوف من كوفيد-19 لاى الطلبة اليمنيين في الصين؟ تم

حساب المتوسطات الحسابية والانحر افات المعيارية للمتغير كما هو مبين في الجدول (2). جدول(2): متوسطات تقديرات عينة البحث

\begin{tabular}{|c|c|c|c|}
\hline مستوى الدرجة & الانحر اف المعياري & 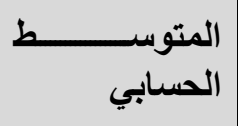 & المتغير \\
\hline مرتفعة & .467 & 2.42 & الخوف من كوفيد-19 \\
\hline
\end{tabular}

من الجدول (2) الموضـح لمتوسطات تقدير ات عينـة البحث، نجد أن مستوى الخوف من كوفيد-19 لدى الطلبة اليمنيين في الصين جـاء بدرجـة مرتفعة، ويعزو الباحث ذلك إلى حالـة الخوف التي تصـاحب انتشـار الأمر اض والأوبئة التي تنتشر بشكل سريع ومفاجئ. تتفق هذه النتيجة مع دراسة (ناجي، 2021) ودراسة . (Mahmud \& Rahman, 2020) . 2. عرض نتائج السؤال الثاني ومناقشتها. وللإجابة على السؤال الثاني من أسئلة البحث الذي نصـه: مـا درجة الثـعور بالوحدة النفسية 19 لدى الطلبة اليمنيين في الصين؟ تم حساب المتوسطات الحسابية والانحر افات المعيارية للمتغير كما هو مبين في الجدول (3). جدول(3): متوسطات تقديرات عينة البحث

\begin{tabular}{|c|c|c|c|}
\hline | مستوى الارجة & 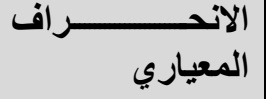 & المتوسط الحسابي & المتغير \\
\hline مرتفعة & .321 & 2.61 & الشعور بالوحدة النفسية \\
\hline
\end{tabular}

من الجدول (3) الموضـح لمتوسطات تقديرات عينـة البحث، نجد أن درجـة الثـعور بالوحدة النفسية لدى الطلبة اليمنيين في الصين جـاء بدرجـة مرتفعة، ويعزو الباحث ذلك إلى حالـة الاغتر اب التي يعيشـها الطالب اليمني في الصبين بعيدًا عن موطنه و أهلـه 
واختلاف البيئة الاجتماعية والثقافية والتعليمية بين البلدين. تتفق هذه مع الدراسة السابقة التي أجر اها الباحث على نفس العينة (الضبياني،

3. عرض نتائج السؤال الثالث ومناقشتها.

وللإجابة على السؤال الثالث من أسئلة البحث الذي نصه: ما علاقة الخوف من كوفيد-19 بالثعور بالوحدة النفسية لاى الطلبة

اليمنيين في الصين؟ تم إجر اء اختبار بيروسون للارتباط، للمتغيرات كما هو مبين في الجدول (4).

جدول(4): اختبار بيروسون للارتباط

\begin{tabular}{|c|c|c|}
\hline مستوى الدلالة & |درجة الارتباط & الخوف من كوفيد-19 \\
\hline .000 & $.753 * *$ & الشعور بالوحدة النفسية \\
\hline
\end{tabular}

من الجدول (4) الموضح لنتيجة اختبار بيروسون للارتباط، نجد وجود علاقة ارتباط طردية قوية بين مستوى الخوف من

كوفيد-19 ودرجة الشعور بالوحدة النفسية، و هذا طبيعي فالوحدة النفسية من أهم المشكلات النفسية التي تتأثر وتؤثر في المشكلات النفسية الأخرى كالقلق و التوتر و الخوف الز ائد والاكتئاب. تتفق هذه النتيجة مع ما نوصلت الى در اسة (عامر، 2020).

4. عرض نتائج السؤال الرابع ومناقثتها.

ولإجابة عن السؤال الر ابع من أسئلة البحث الذي نصده: هل يوجد فروق بين مستوى الخوف من كوفيد-19 ودرجة الثـعور

بالوحدة النفية لاى الطلبة اليمنيين في الصين طبقًا لمتغير النوع الاجتمـاعي (ذكر - أنثى)؟ تم إجر اء اختبار (T) لعينتين مستقلتين

للمتغيرات كما هو مبين في الجدول (5).

جدول(5): اختبار T لعينتين مستقلتين

\begin{tabular}{|c|c|c|c|c|c|c|c|}
\hline الدلائة & قيمة T & درجـــات & الالمعياريــراف & الحسابي & 可 & 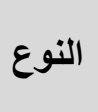 & 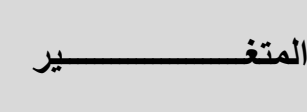 \\
\hline \multirow{2}{*}{.000} & \multirow{2}{*}{37.123} & 299 & .492 & 2.39 & 262 & ذكر & \multirow{2}{*}{ الخوف من كوفيد-19 } \\
\hline & & 40.134 & .421 & 2.37 & 39 & أنثى & \\
\hline \multirow[t]{2}{*}{.000} & \multirow[t]{2}{*}{31.298} & 299 & .385 & 2.42 & 262 & 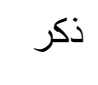 & \multirow[t]{2}{*}{ الثعور بالوحدة النفسية } \\
\hline & & 39.683 & .361 & 2.48 & 39 & أنثى & \\
\hline
\end{tabular}




$$
\text { من الجدول (5) الموضح لنتيجة اختبار (T) لعينتين مستقلنين، نجد ما يلي: }
$$

- يوجد فروق بين استجابات عينة البحث لمستوى الخوف من كوفيد-19 طبقًا لمتغير النوع الاجتماعي لصسالح الذكور ، ويعزي الباحث ذللك إلى طبيعة الأنشطة التي يشـارك فيها الطلبة واحتكاكهم بـالمجمع المحيط والأصدقاء أكثر من الطالبات، الأمر الذي يجعل الطلبة أكثر عرضةً للإصابة بالخوف من كوفيد-19. - يوجد فروق بين استجابات عينة البحث لدرجة الشعور بالوحدة النفسية طبقًا لمتغير النوع الاجتماعي لصالح الإناث، ويعزي الباحث ذلك إلى العزلة الاجتماعية التي تعيشها معظم الطالبات اليمنيات في الغربة بعيدًا عن الأهل والأصدقاء.

\section{المبحث الخامس: الخلاصة والتوصيات}

\section{1}

$$
\text { من النتائج السابقة نجد الآتي: }
$$

- مستوى الخوف من كوفيد-19 ودرجة الثعور بالوحدة النفسية كانا بدرجة مرتفعة.

- توجد علاقة طردية بين مستوى الخوف من كوفيد-19 ودرجة الشعور بالوحدة النفسية.

- توجد فروق في مستوى الخـوف من كوفيد-19 طبقًا لمتغير النوع الاجتماعي لصـالح الذكور وفروق بين استجابات عينـة البحث لارجة الثعور بالوحدة النفسية لصالح الإناث.

- نوصي الجالية اليمنية في الصين و الجهات ذات العلاقة لدعم أنشطة اتحاد طلاب اليمن في الصين الثقافية والاجتماعية والرياضية لما لها من دور في كسر العزلة الاجتماعية والتخفيف من الشعور بالوحدة النفسية لدى الطلبة. - نوصي الملحقية الثقافية بسفارة اليمن في الصين لحل مشكلات الطلبة التي تستجد أولًا بأول؛ لأن تر اكمها أو تأخير النظر فيها يؤدي إلى المزيد من المشكلات النفسية لدى الطلبة.

- نوصي السفارة اليمنية و اتحاد طلاب اليمن في الصين للتعاقد مع مرشد نفسي وتوفير خط ساخن للتو اصل معه من قبل الطلبة الذين قد نواجههم مشكلات نفسية ويحتاجون إلى التوجيه و الإرشاد.

- نوصي اتحاد طلاب اليمن في الصين بإقامة أنشطة وفعاليات تستهدف الطالبات لتعزيز قدر اتهن ومهار اتهن و لا سيما في الجانب الثقافي والاجتماعي. 
- إجر اء در اسات مماتلة تستهدف الطلبة اليمنيين الدار اسين في البلدان الاخرى.

- إجر اء در اسات تطبيقية للتحقق من هذه المشكلات التي يعاني منها الطلبة اليمنيين في الصين ومعالجتها. 


\section{الاستبانة بصورتها النهائية:}

\begin{tabular}{|c|c|c|c|c|c|c|}
\hline & & & & & \multicolumn{2}{|c|}{ استبانة الخوف من كوفيد 19} \\
\hline كبيزة جدًا & كبيرة & متوسطة & قلبلة & قليلة جدًا & \multicolumn{2}{|c|}{ الفقرات } \\
\hline & & & & & أتجنب الخروج من المنزل حتى لا أقابل أحدًا & 1 \\
\hline & & & & & أتجنب الصلاة في جماعة خوفًا من ملامسة الآخرين. & 2 \\
\hline & & & & & أتجنب زيارة أققاربي خوفًا من كورونا. & 3 \\
\hline & & & & & أخاف من الآخرين حتى لا يصييني الفيروس. & 4 \\
\hline & & & & & أخاف أن لا أسلم على أحدًا خوفُا من ملامسة يده. & 5 \\
\hline & & & & & كورونا. مـن شـر اء الحاجـات اليوميـة؛ لأنهـا قـــ تحمـل فيـروس & 6 \\
\hline & & & & & أتجنب ملامسة وجهي خوفًا من الإصابة بكورونا. & 7 \\
\hline & & & & & أخاف أن أصساب من كُورونا؛ خشية الموت. & 8 \\
\hline & & & & & تتنابني أحلام مرعبة في أثناء النوم خوفًا من الإصـابة بكورونا. & 9 \\
\hline & & & & & أنا عندي رعب من الإصـابة بكورونا. & 10 \\
\hline & & & & & أنشك باستمرار أنني أعاني من أعراض كورونا. & 11 \\
\hline & & & & & أشعر بالذعر من سماع الأخبار عن وفيات فيروس كورونا. & 12 \\
\hline
\end{tabular}

\begin{tabular}{|c|c|c|c|c|c|}
\hline & & & & \multicolumn{2}{|c|}{ استبانة الوحدة النفسية } \\
\hline دائمدً & أحيانًاً & نـادرً & أبدًا & \multicolumn{2}{|c|}{ 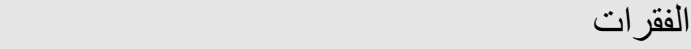 } \\
\hline & & & & أنشعر بانسجام مع الناس من حولي. & .1 \\
\hline & & & & أفتقز إلى الاصدقاء. & .2 \\
\hline & & & & لا يوجد شخص يمكن أن ألجأ إليه. & .3 \\
\hline & & & & لا أشعر بأنني وحيد. & .4 \\
\hline & & & & أشعر بأنني جزء من مجمو عة أصدقاء. & .5 \\
\hline & & & & أشترك بأمور كثيرة مع الناس الذين حولي. & .6 \\
\hline & & & & لم أعد قريبًا من أحد. ل & 7 \\
\hline & & & & لا يشار كني من هم حولي اهتماماتي و أفكاري. & .8 \\
\hline & & & & أنا شخص منفتح على الآخرين. & .9 \\
\hline & & & & هنالك أناس أشعر بأنني قريب منهر. & .10 \\
\hline & & & & أشعر بأن لا أحد يهنم بي. & .11 \\
\hline & & & & علاقاتي الاجتماعية سطحية. & .12 \\
\hline & & & & لا أحد يعرفني حق المعرفة. & 13 \\
\hline & & & & أشعر بالعزلة عن الآخرين. & 14 \\
\hline & & & & أستطيع أن أجد الأصدقاء عندما أريد. & 15 \\
\hline & & & & هناك أناس يفهوونني فعلًا. & 16 \\
\hline & & & & أنشعر أنني غير سعيد كوني منسحبًا كثيرًا. & 17 \\
\hline & & & & الناس حولي لكنهم ليسو ا معي. & 18 \\
\hline & & & & هناك أناس أستطيع التحدث إليهم. & 19 \\
\hline & & & & هنالك أناس أستطيع أن ألجأ إليهم. & 20 \\
\hline
\end{tabular}




\section{قائمة المراجع والمصادر:}

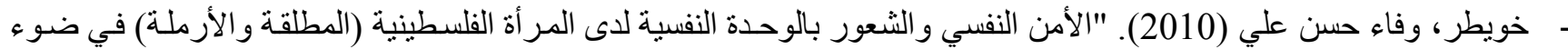

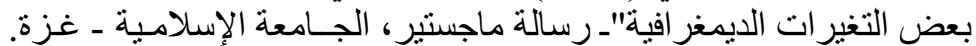

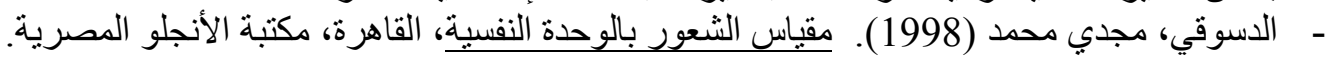

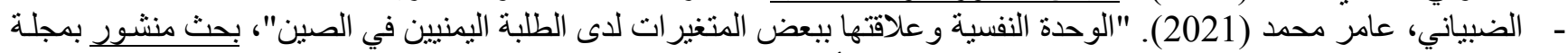

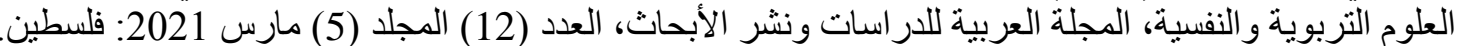

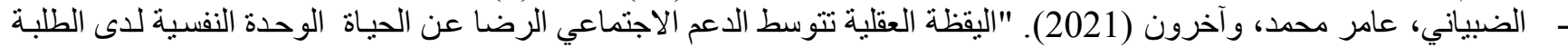

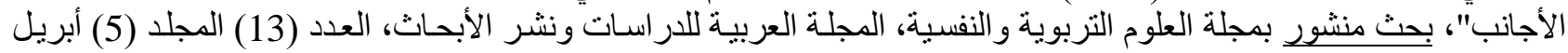

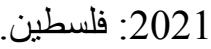
عامر ، عبدالناصر السيد (2021) : عل21) "الخوف من وباء كوفيد-19 لدى الطلبة"، بحث منشور بالمجلة التربوية، جامعة سوهاج، العدد

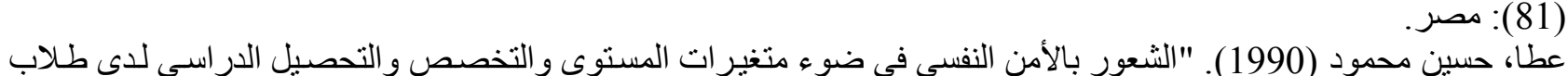

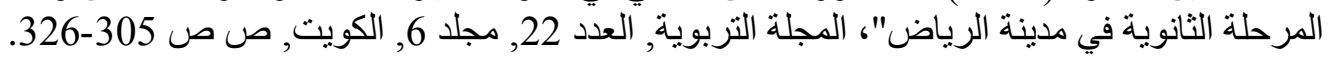

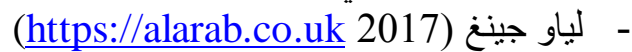

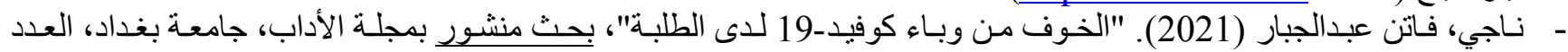
(136)

- .Karaka s, M. Covid-19 salgınının çok boyutlu sosyolojisi ve yeni normal meselesi.Sosyol. Derg.2020,40, 541-573.

- Andersen K.G., Rambaut A., Lipkin W.I., Holmes E.C., Garry R.F. The proximal origin of SARSCoV-2. Nat. Med. 2020;26:450-452. doi: 10.1038/s41591-020-0820-9.

- Arora, A., Jha, A. K., Alat, P., \& Das, S. S. (2020). Understanding coronaphobia. Asian journal of psychiatry, 54, 102384. https://doi.org/10.1016/j.ajp.2020.102384

- Asher, S. \& Julie, A. (2003). Loneliness and peer relations in childhood, current directions in psychological science, Vol. 12, $\mathrm{N}^{\circ}$ (3), 75-78.

- Asmundson G.J.G., Taylor S. Coronaphobia: Fear and the 2019-nCoV outbreak. J. Anxiety Disord. 2020;70 doi: 10.1016/j.janxdis.2020.102196.

- Berg-Weger, M., \& Morley, J. E. (2020). Loneliness in old age: an unaddressed health problem.

- Cacioppo, J. T., Hawkley, L. C., Crawford, L. E., Ernst, J. M., Burleson, M. H., Kowalewski, R. B., ... \& Berntson, G. G. (2002). Loneliness and health: Potential mechanisms. Psychosomatic medicine, 64(3), 407-417.

- Chakraborty K., Chatterjee M. Psychological impact of COVID-19 pandemic on general population in West Bengal: a cross-sectional study. Indian J. Psychiatry. 2020;62:266-272. doi: 10.4103/psychiatry.IndianJPsychiatry.

- Daniel, R.; Kathleen Hall, J. Conspiracy theories as barriers to controlling the spread of COVID-19 in the U.S.Soc. Sci. Med.2020,263, 113356.

- Hawkley, L. C., \& Cacioppo, J. T. (2010). Loneliness matters: A theoretical and empirical review of consequences and mechanisms. Annals of behavioral medicine, 40(2), 218-227.

- Li W., Yang Y., Liu Z.-H., Zhao Y.-J., Zhang Q., Zhang L., Cheung T., Xiang Y.-T. Progression of mental health services during the COVID-19 outbreak in China. Int. J. Biol. Sci. 2020;16:1732-1738. doi: $10.7150 /$ ijbs. 45120

- Lindsay, E. K., Young, S., Brown, K. W., Smyth, J. M., \& Creswell, J. D. (2019). Mindfulness training reduces loneliness and increases social contact in a randomized controlled trial. Proceedings of the National Academy of Sciences, 116(9), 3488-3493.

- Lynch, J. (1977): "The broken heart. The Medical Consequences of Loneliness", New York.

- Madhava Chandran, K.; Valsan, T.; Naveena, K.; Krishnakumar, K. Analysis of the uncertainty among people due to the COVID-19Pandemic.Int. J. Indian Psychol.2020,8, 1894-1901. 
- Melki, J.; Tamim, H.; Hadid, D.; Farhat, S.; Makki, M.; Ghandour, L.; Hitti, E. Media Exposure and Health Behavior duringPandemics: The Mediating Effect of Perceived Knowledge and Fear on Compliance with COVID-19 Prevention Measures.Health Commun.2020.

- Moeller, R. W., \& Seehuus, M. (2019). Loneliness as a mediator for college students' social skills and experiences of depression and anxiety. Journal of adolescence, 73, 1-13.

- Muhammad, S.R. Dialogic Content Analysis of Misinformation about COVID-19 on Social Media in Pakistan.Linguist. Lit. Rev.2020,6, 131-143.

- Orhan Koçak, Ömer Erdem Koçak and Mustafa Z. Younis, The Psychological Consequences of COVID-19 Fear and theModerator Effects of Individuals' Underlying Illness andWitnessing Infected Friends and Family (2021).

- Rokach, A. ; Bauer, N and Orzeck, T. (2003): The experience of Loneliness of Canadian and Czech youth". Journal of adolescence. 26) 267-282.

- Salari, N.; Hosseinian-Far, A.; Jalali, R.; Vaisi-Raygani, A.; Rasoulpoor, S.; Mohammadi, M.; Rasoulpoor, S.; Khaledi-Paveh, B.Prevalence of stress, anxiety, depression among the general population during the COVID-19 pandemic: A systematic review andmeta-analysis.Glob. Health2020,16, 57.

- Sermat, V. (1978). Sources of loneliness. Essence: Issues in the Study of Ageing, Dying, and Death, 2(4), 271-276.

- Tanner C. When coronaphobia turns into agoraphobia: 'I struggle to even go out for a walk' inews. 2020 Retrieved fromhttps://inews.co.uk/news/coronavirus-uk-latest-lockdown-restrictionscoronaphobia-agoraphobia-mental-health-434506, June 6, 2020.

- Thamboo, P. A. (2016). The Effects of a Mindfulness-Based Intervention on Feelings of Loneliness and Ruminative Thinking.

- Wang C., Pan R., Wan X., Tan Y., Xu L., Ho C.S., Ho R.C. Immediate psychological responses and associated factors during the initial stage of the 2019 coronavirus disease (COVID-19) epidemic among the general population in China. Int. J. Environ. Res. Public Health. 2020;17 doi: 10.3390/ijerph17051729.

- Weiss,R.S. (1973): Loneliness: The experience of emotional and social isolation. Cambridge Mass. MIT press 184.

- Yoon E. Harvard Business Review; 2020. Behavioral Trends That Will Reshape Our Post-covid rld.https://hbr.org/2020/05/3-behavioral-trends-that-will-reshape-our-post-covid-world.

- Al-Salem. Manal (2021): Difficulties of Distance Training in the Kingdom of Saudi Arabia from the point of view of Trainers and Female Trainers (A Prospective Study) ,Arid International Journal of Educational and Psychological Sciences ,VOL.2 NO.3, January(pp. 119-135). 